\title{
Matrix metalloproteinase 2 and tissue inhibitors of metalloproteinases regulate human aortic smooth muscle cell migration during in vitro aging
}

\author{
Davide Vigetti, Paola Moretto, Manuela Viola, Anna Genasetti, Manuela Rizzi, \\ Evgenia Karousou, Francesco Pallotti, Giancarlo De Luca, Alberto Passi ${ }^{1}$ \\ Dipartimento di Scienze Biomediche Sperimentali e Cliniche, Università degli Studi dell'Insubria, \\ Varese, Italy
}

ABSTRACT As a direct correlation between aging and the risk of onset of vascular disease has been universally accepted, we prepared an in vitro aging model consisting in sequential passages of human aortic smooth muscle cells (AoSMC) in order to evaluate the cell behavior changes during aging. Because matrix metalloproteinases (MMP) are actively involved in matrix remodeling and disease outcome, in our model we found active MMP-2 only in the conditioned medium of young AoSMCs, whereas aged cells showed only the inactive zymogen form of MMP-2 (pro-MMP2). We ascribed the pro-MMP-2 activation in young cells to an increase in membrane type 1 matrix metalloproteinase (MT1-MMP) content. Furthermore, we found that transcripts coding for tissue inhibitor of metalloproteinases (TIMPs) were up-regulated in aged cells, and this increase of TIMPs could also prevent proMMP-2 activation in aged cells. Moreover, we demonstrated that young AoSMCs possess higher migratory capabilities than aged cells. The young AoSMC migration can be inhibited by adding TIMP-1 and TIMP-2 to the cells reproducing aged AoSMC migratory behavior. Finally, the role of MMP-2 and TIMP-2 in AoSMC migration was confirmed silencing MMP-2 and TIMP-2 in young and aged AoSMCs, respectively; therefore, in this study we showed that these enzymes play a pivotal role in the regulation of the AoSMC migration during in vitro aging.-Vigetti, D., Moretto, P., Viola, M., Genasetti, A., Rizzi, M., Karousou, E., Pallotti, F., De Luca, G., Passi, A. Matrix metalloproteinase 2 and tissue inhibitors of metalloproteinases regulate human aortic smooth muscle cell migration during in vitro aging. FASEB J. 20, 1118-1130 (2006)

Key Words: senescence $\cdot$ extracellular matrix $($ ECM) $\cdot$ siRNA - gene expression

Smooth MUScle cells (SMCs) are mainly located within the tunica media of blood vessels in a contractile state, expressing specific genes essentials for SMC functions, such as regulation of vessel tone and blood pressure (1). Under pathological conditions, such as vessel injury or atherosclerotic plaque development,
SMCs become exposed to certain growth factors, inflammatory cytokines, and other circulating components that induce a transformation from the contractile to a synthetic state (2). In the latter state only do SMCs acquire the ability to proliferate, migrate, and accumulate within the intimal layer of arteries under the influence of chemotaxis-inducing growth factors. During migration, SMCs have been shown to digest major extracellular barriers such as basal membranes, interstitial collagens, and proteoglycans (3-5). This enzymatic breakdown of ECM components is mediated by the tightly regulated activity of proteases $(6,7)$. Within this group of proteases, the family of matrix metalloproteinases (MMPs) is essential for the digestion of ECM components such as collagens, gelatins, or proteoglycans $(5,9)$. In this process, MMPs can release growth factors bound to specific proteoglycans (i.e., heparan sulfate proteoglycan can bind FGF-2 activity) (9), thus regulating the viability of factors involved in SMC migration and in the pathological response of surrounding cells (10).

The two gelatinases MMP-2 and MMP-9 have received particular attention in the analysis of vascular remodeling and, at least in vitro, both enzymes have very similar substrate specificity. MMP-2 has been demonstrated to degrade native type IV, V, VII, and X collagen, as well as denaturated collagens, proteoglycans, elastin, and growth factors (8). Expression of the two gelatinases in the vascular wall is strictly controlled; MMP-2 shows a basal expression in the media, whereas MMP-9 expression is detectable after injury or inflammatory stimulation (11). However, MMP-2 has been shown to possess an altered synthesis and activation during vessel injury and cytokine treatment (12).

The synthesis and activation of MMPs constitute a well-controlled process in which zymogen, the inactive form of the MMPs, is cleaved to produce the active form. In this process, membrane type 1 metalloprotein-

\footnotetext{
${ }^{1}$ Correspondence: Dipartimento di Scienze Biomediche Sperimentali e Cliniche, Università degli Studi dell'Insubria, via J.H. Dunant 5, Varese 21100, Italy. E-mail: alberto.passi@ uninsubria.it

doi: 10.1096/fj.05-4504com
} 
ase (MT1-MMP) complex plays a pivotal role in the specific activation of pro-MMP-2 (13). On the other hand, tissue inhibitors of metalloproteinases (TIMPs) can interact with active MMPs to inhibit their action. In fact, it has been demonstrated that TIMP-1 and TIMP-2 are the main proteins involved in such processes (14, 15). Moreover, some investigators have suggested a role for TIMPs in preventing morphological modifications of the vascular structures during degenerative diseases (16-18).

It is known that the risk for atherosclerosis or other vascular diseases correlates with mutated proliferative and migratory properties of SMCs during aging (1921). Moreover, biochemical analyses of normal vs. altered vessels show several modifications of ECM components (22) that clearly involved alterations of the enzymes responsible for ECM synthesis and/or degradation. In fact, in thick intima of aged rats MMP-2 is increased (23), and mice with genetic deletions of MMP-2 show a decreased intima thickness compared to wild-type animals (6), indicating the important role of this enzyme in such phenomenon.

Previous observations reported that an altered expression of enzymes regulating the ECM turnover are implicated in vascular diseases (22); here we describe that a fine regulation of MMP-2 and TIMPs can modulate the migration of human aortic SMCs (AoSMCs) during in vitro aging.

\section{MATERIALS AND METHODS}

\section{Cell culture}

Human primary aortic smooth muscle cells (AoSMCs, donor age 17, male) were purchased from Cambrex and grown in SmGm2 complete culture medium (Cambrex, Baltimore, MD, USA) supplemented with 5\% FBS. This cell culture medium was used in all the experiments. The cultures were maintained in an atmosphere of humidified $95 \%$ air $/ 5 \% \mathrm{CO}_{2}$ at $37^{\circ} \mathrm{C}$. Whenever the culture became nearly confluent, the cells were trypsinized, counted using trypan blue exclusion, and subcultured at a density of 3500 cells $/ \mathrm{cm}^{2}$. Population doublings (PD) were determined at each passage and calculated as follows: $\mathrm{PD}=\log$ (number of cells obtained/initial number of cells) $/ \log 2$. In the experiments 4 PD and $18 \mathrm{PD}$ cells were used and referred as young and aged AoSMCs, respectively. The senescent phenotype was confirmed by $\beta$-galactosidase activity assay (Calbiochem, San Diego, CA, USA) as described (24) and p16 $6^{\text {INK4a }}$ (hereafter p16) mRNA expression (25).

\section{Immunofluorescence}

Young and aged AoSMCs grown on coverslips were rinsed with PBS and fixed in methanol. The coverslips were preincubated with PBS containing 2\% FBS and incubated with a solution containing anti- $\alpha$ smooth muscle actin ( $\alpha$-SMA) monoclonal antibodies (Clone 1A4, Sigma; 1:1000) in PBS containing $2 \% \mathrm{FBS}$ for overnight at $4^{\circ} \mathrm{C}$. The coverslips were washed with PBS, then incubated with a solution containing fluorescein conjugated anti-mouse immunoglobulin $(\mathrm{H}+\mathrm{L})$ (1:500) in PBS containing 2\% FBS. Polymerized actin filaments were detected on incubating paraformaldehyde fixed cells with rhodamine-conjugated phalloidin (Molecular Probes, Eugene, OR, USA; dilution 1:40). Fluorescence images were obtained using Delta Vision deconvolution system.

\section{Quantitative RT-PCR}

Total RNA from both young and aged AoSMCs was extracted using Trizol reagent (Invitrogen, San Diego, CA, USA) following the manufacturer's protocol. Total RNA from normal fetal aorta (Stratagene, San Diego, CA, USA) and total RNA from adult aorta (Ambion, Austin, TX, USA) were used to detect the gene of interest in in vivo conditions. To remove DNA contamination, DNase (Ambion) treatment was performed in all samples. One $\mu \mathrm{g}$ of total RNA was retrotranscribed using high capacity cDNA synthesis kit (Applied Biosystems, Foster City, CA, USA) for $2 \mathrm{~h}$ at $37^{\circ} \mathrm{C}$.

Quantitative real-time RT-PCR was performed on Abi Prism 7000 instrument (Applied Biosystems) using Taqman Universal PCR Master Mix (Applied Biosystems) following manufacturer's instructions. Probe and primers were from developed TaqMan gene expression assay reagents (Applied Biosystems). The following TaqMan gene expression assays were used: MMP-2 (Hs00234422), MMP-9 (Hs00234579), TIMP-1 (Hs00171558), TIMP-2 (Hs00234278), $\alpha-S M A$ (Hs00426835), calponin (Hs00154543), p16 (CDKN2A; Hs00233365), MT1-MMP (also known as MMP14, Hs00237119), and RNAseP (Hs00706565). Fluorescent signals generated during PCR amplifications were monitored and analyzed with Abi Prism 7000 SDS software (Applied Biosystems). Comparison of the amount of each gene transcript among different samples was made using RNAseP as reference. Standard curves were generated by serial dilution of cDNA and the relative quantitative evaluation of target gene levels was performed by comparing $\Delta$ Cts (Applied Biosystems user bulletin number 2).

\section{Zymography}

To detect MMP-2 and MMP-9 activities, conditioned medium from young and aged AoSMCs was electrophoresed on SDSpolyacrylamide gels containing $1 \mathrm{mg} / \mathrm{ml}$ gelatin. Samples were loaded in the gels without denaturization in the presence of reducing agents. Gel was washed at room temperature for $2 \mathrm{~h}$ in $2.5 \%$ Triton $\mathrm{X}-100$ and incubated overnight at $37^{\circ} \mathrm{C}$ in $10 \mathrm{mM} \mathrm{CaCl}_{2}, 150 \mathrm{mM} \mathrm{NaCl}$, and $50 \mathrm{mM}$ Tris-HCl, pH 7.5 buffer. The gel was stained in $2 \%$ (v/v) Coomassie blue G-250 and photographed on a light box after appropriate destaining. Proteolysis was detected as a white zone in a dark blue field.

\section{Quantification of MMP-2}

The quantification of MMP2 was done by using the ELISA kit Matrix Metalloproteinase-2 Biotrack Activity Assay System (Amersham Biosciences, Little Chalfont, UK) following the manufacturer's instruction. To determine total MMP-2 activity (i.e., pro- plus active MMP-2), samples were treated with p-aminophenylmercuric acetate (APMA), then tested with the ELISA kit reported above. Plates were read in a Biotrack ELISA reader (Amersham) at $405 \mathrm{nM}$.

\section{Reverse zymography}

For detection of TIMP-1 and TIMP-2 activities, reverse zymography procedures were carried out as described by Oliver et al. (26). Briefly, young and aged AoSMC conditioned medium was concentrated 10-fold using Amicon filter systems 
(3000 Da cutoff) and loaded onto a $15 \%$ polyacrylamide gel containing $15 \mathrm{mg} / \mathrm{ml}$ gelatin and $50 \mathrm{ng} / \mathrm{ml}$ active recombinant human MMP-2 (Calbiochem) without denaturing and reducing agents. Normalization of sample loading on each gel was obtained by application of an equal volume of cell conditioned media standardized to total cell number. After electrophoresis, gels were rinsed in $2.5 \%$ Triton X-100 to remove SDS and incubated overnight at $37^{\circ} \mathrm{C}$ in $10 \mathrm{mM}$ $\mathrm{CaCl}_{2}, 150 \mathrm{mM} \mathrm{NaCl}$, and $50 \mathrm{mM}$ Tris-HCl, $\mathrm{pH} 7.5$ buffer. Subsequently gels were stained with Coomassie blue G-250 and destained with $30 \%$ methanol $10 \%$ glacial acetic acid solution. The presence of TIMPs in the samples inhibits MMP-2 activity visualizing dark bands on a clear background.

The reverse zymography procedure was also used to estimate the TIMPs activity in cell membrane protein extracts that was prepared scraping AoSMCs into cells were washed with PBS and scraped into ice-cold lysis buffer $(20 \mathrm{mM}$ Tris-HCl, pH 7.4, $150 \mathrm{mM} \mathrm{NaCl)}$ containing protease inhibitory cocktail (Roche, Nutley, NJ, USA) and lysed by sonication. The extract was centrifuged at $15,000 \mathrm{~g}$ for $10 \mathrm{~min}$ at $4^{\circ} \mathrm{C}$ and the pellet was re-extracted in lysis buffer supplemented with $0.5 \%$ Triton $\mathrm{X}-100$ on ice, yielding the membrane fraction. Protein concentrations were determined using the Bradford method (Bio-Rad, Hercules, CA, USA) as described by the supplier, with BSA as a standard.

\section{Migration assay}

Cell migration assay was performed using Transwell (Costar, Cambridge, MA, USA) system, which allows cells to migrate throughout a $8 \mu \mathrm{m}$ pore size polycarbonate gelatin-coated membrane. Briefly, Transwell gelatin coating was performed in $0.5 \%$ gelatin for $2 \mathrm{~h}$ at $37^{\circ} \mathrm{C}$ and drying overnight at room temperature. Cells were trypsinized, washed, and resuspended in serum-free SmGm2. $1 \times 10^{5}$ cells were added to the upper chamber of Transwells. The lower chamber was filled with $1.5 \mathrm{ml}$ serum-free $\mathrm{SmGm} 2$ or $1.5 \mathrm{ml}$ serum-free SmGm2 containing $0.3 \mu \mathrm{g} / \mathrm{ml}$ TIMP-1 (Calbiochem), or 0.3 $\mu \mathrm{g} / \mathrm{ml}$ TIMP-2 (Calbiochem) or $5 \mu \mathrm{g} / \mathrm{ml}$ GM6001 (a chemical inhibitor of MMPs purchased from Chemicon, El Segundo, CA, USA), or $0.3 \mu \mathrm{g} / \mathrm{ml}$ active-MMP-2 (Calbiochem). After $6 \mathrm{~h}$, filters were removed and cells remaining on the upper surface of the membrane were removed with a cell scraper. Membranes were then washed with PBS, and cells present beneath the membrane were fixed with cold methanol for 15 min and stained with $0.2 \%$ Crystal violet. Cell count was performed under a light microscope (magnification $20 \times$ ) considering 6 high-power unrelated fields (HPF). Triplicate independent experiments were performed.

\section{Western blot}

Young and aged AoSMC conditioned medium was concentrated 10-fold using Amicon filter systems (3000 Da cutoff). To detect MMP-2, the concentrated medium was further purified using gelatin-Sepharose (Amersham Pharmacia Biotech) (27). Normalization of protein loading on each blot was obtained by application of an equal volume of cell conditioned media standardized to total cell number. After SDSPAGE under reducing conditions, proteins were blotted onto PVDF membrane (Amersham Bioscience). After transfer, the blots were stained with Ponceau S (Sigma Chemical Co., St. Louis, MO, USA) to confirm equal loading. Membranes were probed with polyclonal anti-MMP-2 antibodies (IM33T, Calbiochem) or anti-TIMP-2 monoclonal antibody (mAb) (IM82T, Calbiochem). Signals were revealed using secondary peroxidase-conjugated antibodies and the band visualization was carried out by the chemiluminescence method described by Amersham Pharmacia Biotech.

To detect MT1-MMP, cell extracts were prepared from young and aged AoSMCs by means of radio-immuno-precipitation assay buffer (50 Mm Tris-HCl pH 7.4, $150 \mathrm{mM} \mathrm{NaCl}$, $1 \%$ (v/v) Igepal CA-630 (Sigma, I-3021) supplemented with protease inhibitor cocktail (Roche)) and anti-MT1-MMP mAb (Calbiochem) was used as described above. As a control for protein loading and for normalization, $\alpha$-tubulin was also revealed on MT1-MMP blots using a mAb purchased from Sigma.

\section{MMP-2 and TIMP-2 silencing}

Small interfering RNA (siRNA) was adopted to reduce MMP-2 and TIMP-2 expression in young and aged AoSMCs, respectively. MMP-2 validated siRNA kit (code 51191), TIMP-2 predesigned siRNA (code 118689) and negative control siRNA \#1 kit (scramble, code 4611) were purchased from Ambion and used as recommended. Transfections were performed using Nucleofector apparatus (Amaxa, Gaithersburg, MD, USA) and Human AoSMC (aortic smooth muscle cells) Nucleofector ${ }^{\mathrm{TM}}$ Kit with $5 \mu \mathrm{g}$ of either MMP-2 siRNA, TIMP-2 siRNA, or scramble siRNA. Two micrgrams of Green fluorescent protein (GFP) plasmid (Amaxa) was used to test the transfection efficiency. After $48 \mathrm{~h}$ of incubation, half of the transfected cells were used for migration experiments. Total RNA was extracted from the remaining cells and the reduced MMP-2 expression was checked by real-time PCR. Conditioned cell medium was used in gel zymography experiments to evaluate the remaining MMP-2 activity after siRNA transfections.

\section{Statistical analyses}

Unpaired Student's $t$ test was performed for statistical analyses using Origin 7.5 software (Microcal Software, Studio City, CA, USA). Experiments were performed in triplicate and data are expressed as the mean $\pm \mathrm{SE}$.

\section{RESULTS}

\section{Identification of gelatinase activities in young and aged AoSMCs}

We set up an in vitro aging model in which commercial AoSMCs (Cambrex) have been cultured in completeSmGm2 medium (Cambrex) for several population doublings (PD). We consider as "young" the cells with 4 PD after thawing, and as "aged" the cells with 18 PD after thawing. The cytochemical senescent phenotype was examined by using senescence-associated $\beta$-galactosidase (SA $\beta$-Gal) activity, which has been shown to be a marker of senescence in vascular cells as well as in fibroblasts (28). As shown in Fig. $\mathbf{A}, \boldsymbol{B}$, almost all aged AoSMCs revealed SA $\beta$-Gal activity, while only a few young cells were stained in cultures at lower PD. Moreover, the enlarged size of 18 PD AoSMCs is typical of aged (near to senescence) cells (28).

We have evaluated p16 ${ }^{\text {INK4a }}$ (hereafter p16) another cell senescence marker (29) unrelated to SA $\beta$-Gal activity. We quantified the p16 mRNA, which was recently described to increase its stability during repli- 


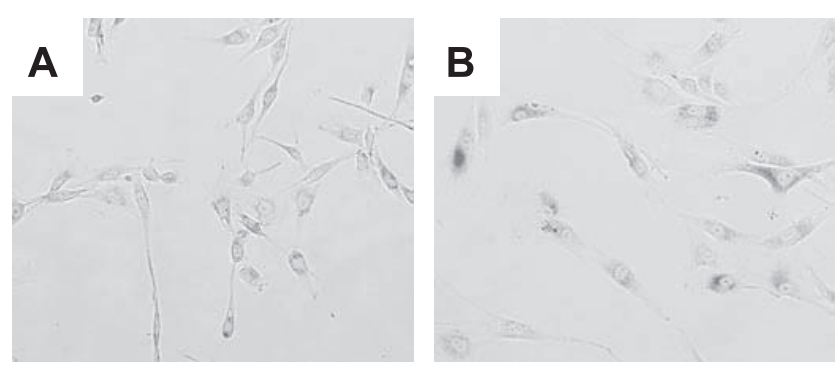

SA- $\beta-G A L$

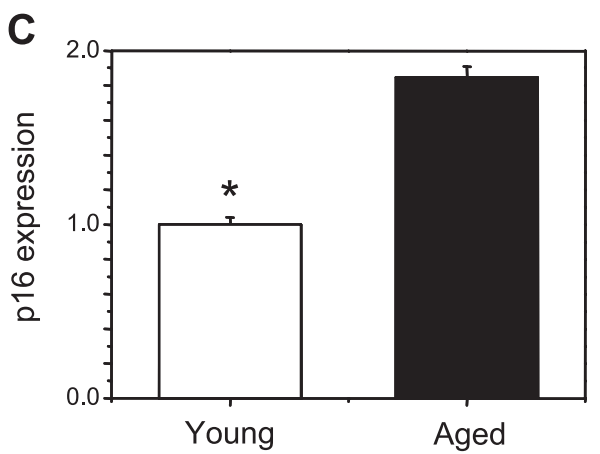

Figure 1. AoSMCs during in vitro aging express senescence markers. $A, B)$ Detection of SA- $\beta$-gal activity in young $(A)$ or aged $(B)$ AoSMCs using phase contrast microscope (Olympus) at the same magnification with a $20 \times$ objective. $C$ ) Quantitative real-time RT-PCR quantification of p16 cDNA in young and aged AoSMCs. $* P<0.01$ aged vs. young. Relative expression is expressed in arbitrary units.

cative senescence (25). We have performed quantitative real-time RT-PCR using the TaqMan gene expression assay developed by Applied Biosystems and, as shown in Fig. $1 C$, a clear and statistically significant accumulation of p16 transcript was detected in aged AoSMCs, confirming that 18 PD AoSMCs can be considered "aged" expressing the typical marker of senescent cells.

To verify that the numerous culture passages did not affect the AoSMC phenotype, we have considered $\alpha$-SMA and calponin, well-known markers of SMC. By means of quantitative real-time RT-PCR, we detected that all the above cited markers were expressed at the same levels both in young and aged AoSMCs (results not shown). Moreover, immunolocalizations and Western blot analyses were performed to quantify and visualize the $\alpha$ SMA (Fig. $2 A, B, G$ ), and no substantial differences between young and aged AoSMCs were detected. To further confirm this result, we visualized polymerized actin by means of phalloidin conjugated with rhodamine (30). As shown in Fig. 2C-F, the typical SMC actin filaments were detected in both young and aged AoSMCs. Moreover, the same cellular distribution of such filaments revealed that no dedifferentiation events occurred during subsequent cell passages.

To analyze the gelatinase activities secreted into the cell medium of young and aged AoSMCs, we have harvested the conditioned culture medium and loaded onto a zymography gel. As shown in Fig. $3 \mathbf{A}$, both young and aged cells produced a $72 \mathrm{kDa}$ band of gelatinase activity corresponding to the purified zymogen of matrix metalloproteinase 2 from Sigma (pro-MMP-2). Surprisingly, the $\sim 66 \mathrm{kDa}$ band corresponding to the fully activated MMP-2 is detectable only in the conditioned cell culture medium of young AoSMCs. No other gelatinase activity bands were observed on the gel, suggesting that the other well-known MMP-9 is not present in a detectable amount.

To quantify gelatinase activity, we performed MMP-2 ELISA tests and found that the total gelatinase activity (i.e., pro- plus active MMP-2) was $18.7 \mathrm{ng} / \mathrm{ml}$ and 18.1 $\mathrm{ng} / \mathrm{ml}$ in conditioned culture medium of young and aged AoSMCs, respectively. Accordingly with the zy-
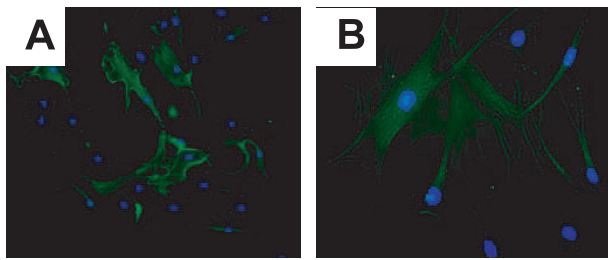

$\alpha$ SMA
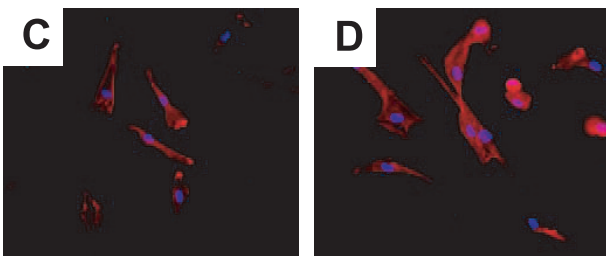

PHALLOIDIN
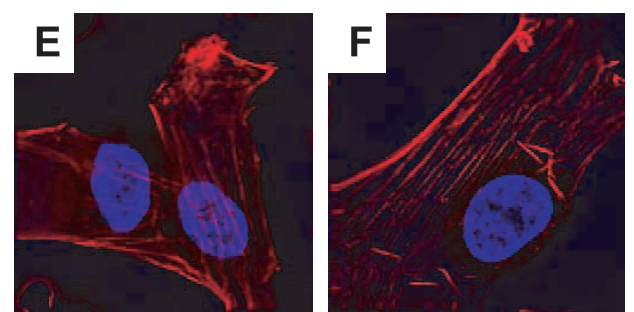

PHALLOIDIN

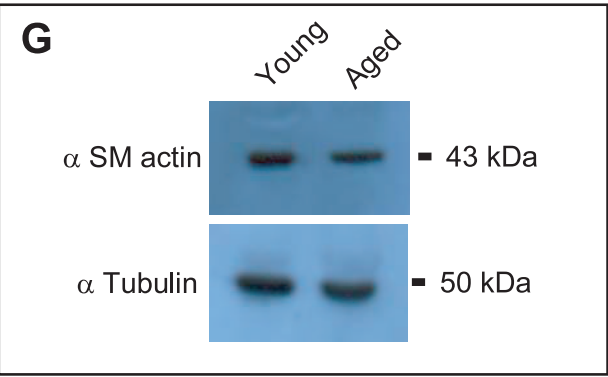

Figure 2. AoSMCs during in vitro aging maintain smooth muscle phenotype. $A, B)$ Immunolocalization of $\alpha$-SMA (green) in young $(A)$ and aged $(B)$ AoSMCs. Nuclei are stained with DAPI (blue). The same magnification (40X objective) were used for both the photomicrographs. $C-F$ ) Localization of actin filaments by rhodamine conjugated phalloidin (red) in young $(C-E)$ and aged $(D-F)$ AoSMCs. Nuclei are stained with DAPI (blue). The same magnification (40× objective) was used for panels $C, D$ in both photomicrographs. Deconvolved images acquired by Delta Vision system are shown in panels $E, F$ (with $100 \times$ objective) for young and aged AoSMCs, respectively. G) A Western blot experiment showing $\alpha$-SMA in cell extract of young and aged AoSMCs. To standardize protein loading, $\alpha$ tubulin was also detected. 

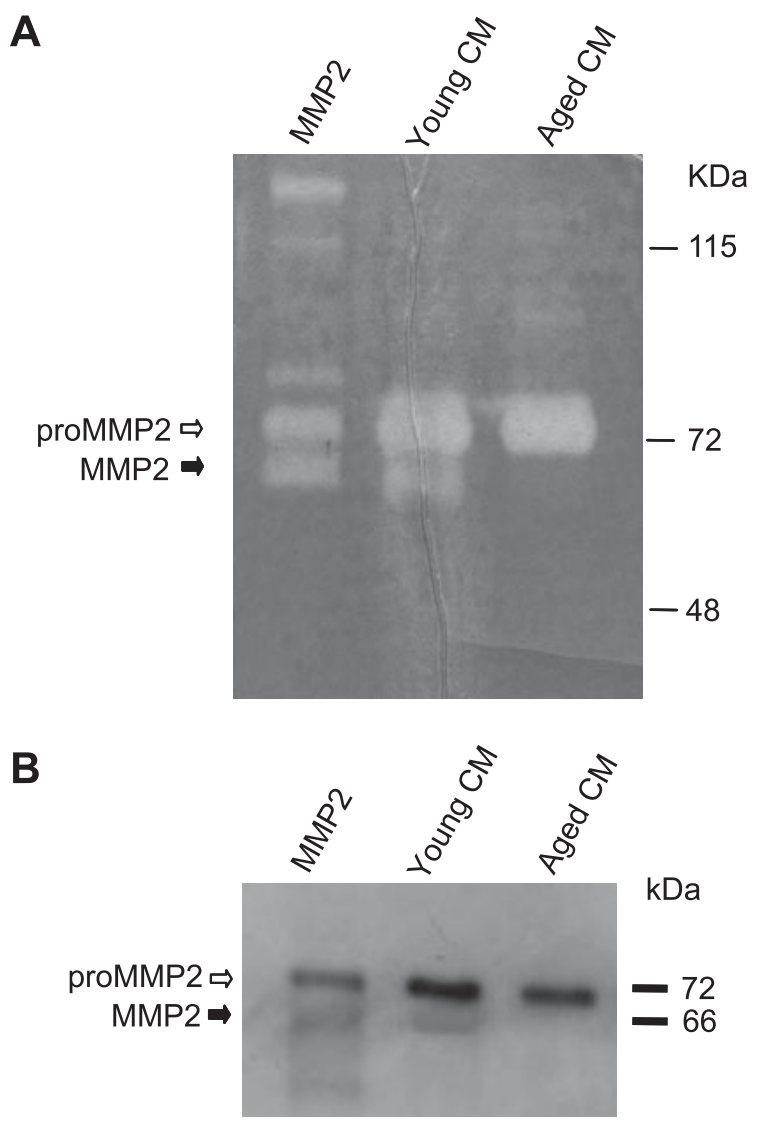

Figure 3. Gelatinase activities during in vitro aging of AoSMCs. A) Zymography analysis. Fifteen microliters of conditioned medium (CM) deriving from $10^{6}$ of either young or aged AoSMCs were loaded onto gelatin-polyacrylamide gel as stated in Materials and Methods. Standard lane contains both active and latent form of MMP-2 (Sigma) and was used as reference. The data shown are representative of two separate experiments. B) Western blot analysis of MMP-2. Concentrated and gelatin-Sepharose purified conditioned medium from young and aged AoSMCs (deriving from the same amount of cells) were electrophoresed and blotted on PVDF membranes and incubated with anti-MMP-2 antibodies. MMP-2 lane is loaded with both latent and active MMP-2 (Sigma).

mography experiment, active MMP-2 (i.e., without chemical activation of pro-MMP-2 in MMP-2 with APMA) in the conditioned culture medium of young AoSMC was $7.5 \mathrm{ng} / \mathrm{ml}$ in young, whereas in aged AoSMC culture, medium was below the detection limit of the kit (i.e., 0.2 $\mathrm{ng} / \mathrm{ml})$.

To identify the gelatinase bands, we have performed Western blot analyses using concentrated conditioned cell culture medium of young and aged AoSMCs with anti-MMP-2 antibodies. As MMP-2 was expressed at low concentration to be visualized without concentration, before the SDS-PAGE separation we performed another affinity purification step using gelatin-Sepharose as outlined by Mazzieri et al. (27). As shown in Fig. 3B, a $72 \mathrm{kDa}$ band corresponding to pro-MMP-2 is present in similar amounts both in young and aged AoSMC conditioned medium. On the other hand, the $66 \mathrm{kDa}$ band, corresponding to the active form of MMP-2, is visualized only in young conditioned medium as expected from zymography.

\section{Quantification of the pro-MMP-2 activator}

As one of the major activator of pro-MMP-2 in physiological conditions is membrane type 1 MMP (MT1MMP) (13), we have quantified its synthesis in young and aged AoSMCs. By quantitative real-time RT-PCR, we have found that the concentration of mRNA coding for MT1-MMP was reduced by $\sim 60 \%$ in aged vs. young AoSMCs (Fig. 4A). We also detected MT1-MMP at protein concentrations by Western blot. MT1-MMP is synthesized as a $65 \mathrm{kDa}$ proenzyme, which is activated by proteolytic cleavage producing the $54 \mathrm{kDa}$ enzyme. Another proteolytic cleavage converts the active enzyme into a $45 \mathrm{kDa}$ inactive protein (13). Using a monoclonal anti-MT1-MMP antibody, we showed that the active MT1-MMP was present in both young and aged AoSMC extracts, even if this band was more intense in young than aged cells (Fig. $4 B$ ).

As MT1-MMP activates pro-MMP-2 through the formation of a complex with TIMP2, we quantified the cellular TIMP2 performing reverse zymography experiments on total protein extracts. As shown in Fig. $4 C$, TIMP2 was present at a higher concentration in young than in aged AoSMCs.

\section{Quantification of MMPs and TIMPs during AoSMC aging}

To better define MMPs expression during in vitro aging of AoSMCs, we quantified the transcripts coding for MMPs by quantitative real-time RT-PCR. Figure $\mathbf{5} \boldsymbol{A}$ shows the relative expression of MMP-2 and MMP-9 mRNA normalized for the content of RNAseP (housekeeping gene). Because the activities of MMP-2 and MMP-9 are strongly regulated by tissue inhibitor of metalloproteinases (TIMP), we also quantified the transcripts coding for such proteins (named TIMP-1 and TIMP-2). As reported in Fig. $5 \mathrm{~A}$, the quantity of MMP-2 mRNA is not significantly different from young to aged AoSMCs, whereas, as expected from the zymography experiment, no MMP-9 transcripts were detectable. The concentration of TIMP-1 and TIMP-2 mRNAs varied during aging, and their amounts show a significant increase in aged AoSMCs $(P<0.001)$.

To visualize TIMP activities present in the conditioned cell culture medium of AoSMCs, we performed reverse zymography analyses. Young and aged AoSMC conditioned medium contained inhibitory bands corresponding to TIMP activities. The 31 and $21 \mathrm{kDa}$ bands corresponded to TIMP-1 and TIMP-2 proteins, respectively (results not shown). Besides these two gelatinase inhibitory activities, two bands at $\sim 23 \mathrm{kDa}$ are present in the conditioned cell culture medium and could be attributed to the glycosylated and unglycosylated forms of TIMP3, which is not directly correlated in MMP-2 activity regulation (8).

Western blot analyses were carried out to better 
A

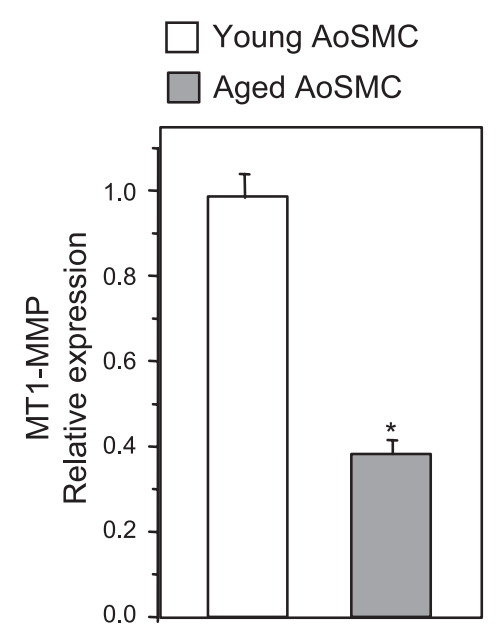

B
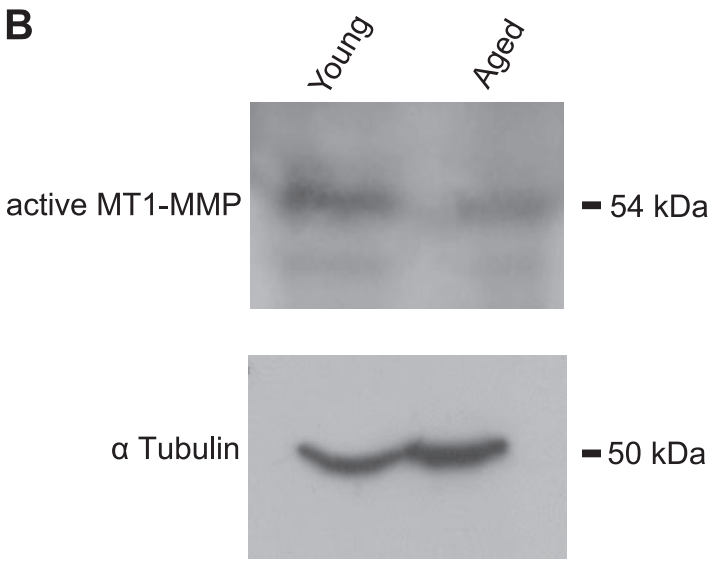

C

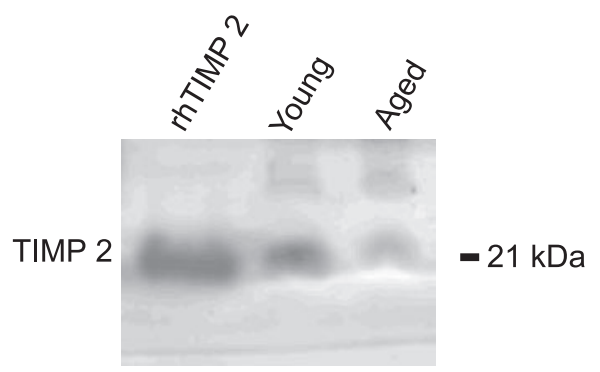

Figure 4. MT1-MMP expression in young and aged AoSMCs. A) Quantification of the transcript coding for MT1-MMP by real-time RT-PCR in young and aged AoSMCs. Relative expression in arbitrary units. $* P<0.01$ young vs. aged. B) Western blot analysis of MT1-MMP. $20 \mu \mathrm{g}$ of cell lysates from young and aged AoSMCs were electrophoresed and blotted on PVDF membranes, and incubated with anti-MT1-MMP antibodies. As loading control, $\alpha$ tubulin has been detected. C) Reverse zymography experiment performed on $10 \mu \mathrm{g}$ of membrane extracted proteins from young and aged AoSMCs showing TIMP2 activity associated to cell extracts. Recombinant human TIMP2 (rhTIMP2) was loaded as a reference.

visualize the presence of TIMP-2 in young and aged AoSMC conditioned medium. As shown in Fig. 5B, a band of $21.0 \mathrm{kDa}$ corresponding to TIMP-2 is visible in both conditioned media in amounts related to the mRNA expression pattern. The recombinant human TIMP-2 (rh-TIMP-2, Calbiochem) used as control in the
Western blot and reverse zymography experiments migrated as a $24.0 \mathrm{kDa}$ protein in reducing gels (Fig. $5 B$ ) and as a $21 \mathrm{kDa}$ protein in nonreducing gels (result not shown), as expected from the rh-TIMP-2 characterization performed by the manufacturer.

To determine whether our in vitro cell aging system could be representative of the gene expression present in in vivo conditions, we performed quantitative realtime RT-PCR on normal aorta RNA prepared from healthy donors of different ages. As a young sample, we used a pool of 5 different fetal aorta RNAs purchased from Invitrogen; as an adult sample we used a pool of 5 different aorta RNAs from donors of $>65$ years old (Ambion). The results reported in Fig. 6 clearly indicate that MT1-MMP, TIMP1 and TIMP2 mRNA levels correlate well with that obtained from AoSMC gene
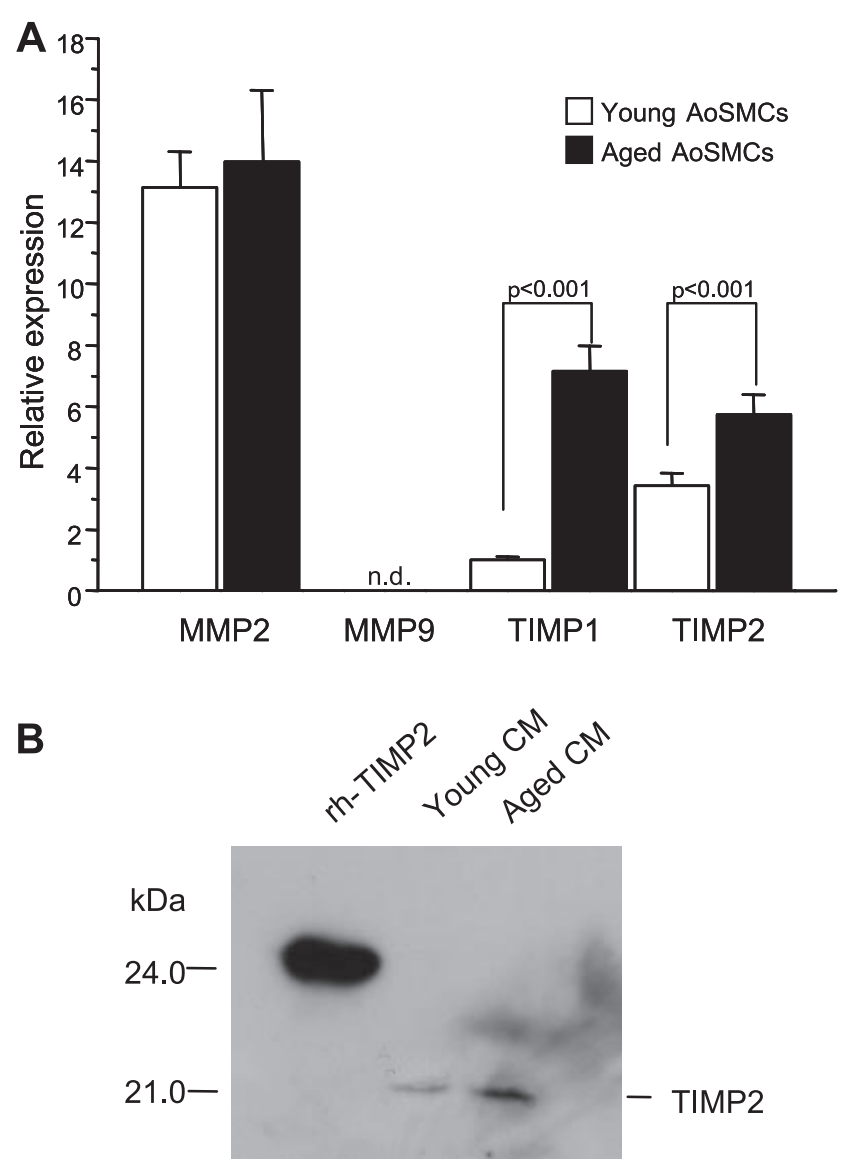

Figure 5. Quantitative analysis of MMPs and TIMPs during AoSMC aging. A) Quantification of the transcripts coding for MMP-2, MMP-9, TIMP-1, and TIMP-2 by real-time RT-PCR in young and aged AoSMCs. Relative expression is expressed in arbitrary units. No statistical difference is observable in the quantity of MMP-2 mRNA in young or aged AoSMCs. Transcripts coding for MMP-9 were not detectable (n.d.). B) Reverse zymography analysis indicating the presence of TIMP activities in concentrated young and aged AoSMCs conditioned media (CM). Recombinant human TIMP-1 (rhTIMP1) and recombinant human TIMP-2 (rh-TIMP2) were used as standard. C) Quantification of TIMP2 by Western blot analysis in $10 \times$ concentrated young and aged AoSMC conditioned medium using anti-TIMP-2 antibodies. One representative reverse zymogram and Western blot experiments are shown. 


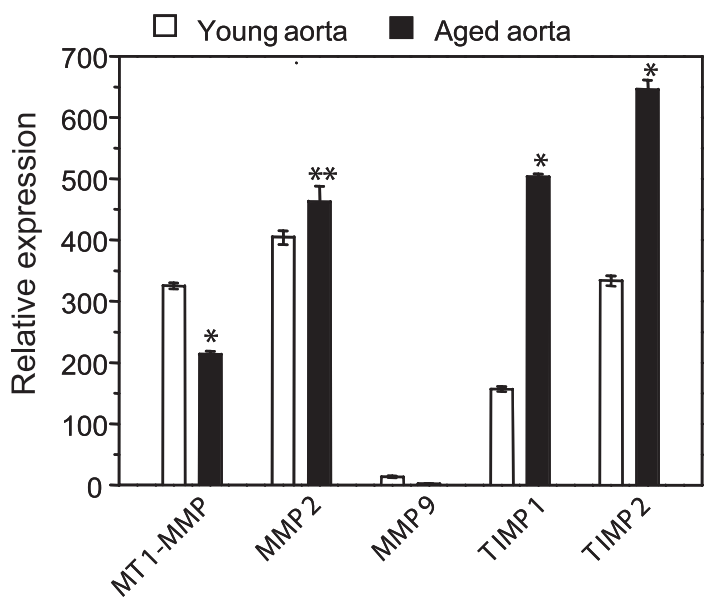

Figure 6. Quantitative analysis of transcripts coding for MT1MMP, MMPs, and TIMPs in in vivo conditions. Quantification of the transcripts coding for MT1-MMP, MMP-2, MMP-9, TIMP-1, and TIMP-2 by real-time RT-PCR in cDNA derived from fetal and aged aorta. Relative expression is expressed in arbitrary units. $* P<0.01$ young vs. aged; $* * P<0.05$ young vs. aged.

expression experiments (Fig. 5A) whereas MMP2 transcript seems to be expressed at higher levels in aged aorta.

\section{MMP-2 and TIMPs modulate AoSMC migration during aging}

Metalloprotease activity is strongly implicated in cell migration, control of ECM degradation, and the release of proteoglycan bound growth factors. Therefore, we have supposed that young AoSMCs could exhibit a migratory behavior since these cells possess active MMP-2 and low TIMP expression. To test this hypothesis, we performed a migration assay using gelatincoated Transwell apparatus (Costar). Figure 7A shows one HPF photograph that clearly demonstrates that young AoSMCs migrate at a higher rate than aged AoSMCs. The same result is shown in Fig. $7 B, C$ comparing the young and aged control bars of the graph.

We have added to the lower chamber of the Transwell apparatus human purified TIMP-1 and TIMP-2 (Calbiochem) to test the possibility that TIMP-1 and TIMP-2 expression could modify the migration of AoSMCs (Fig. 7B, C). The addition of these inhibitory proteins reduced significantly the migration of young AoSMCs $(P<0.05$ and $P<0.001$ for TIMP- 1 and TIMP-2, respectively). Moreover, the addition of GM6001, a chemical inhibitor of MMPs, greatly reduced the migration of both young and aged AoSMCs. On the other hand, the addition of human purified MMP-2 (Calbiochem) stimulated the migration of young AoSMCs and, to a lesser extent, that of aged AoSMCs.

We have also silenced the MMP-2 expression in young AoSMCs by using siRNA, thus verifying the specificity of MMP-2 related migratory behavior. The use of Nucleofector apparatus (Amaxa) and the human primary AoSMC Nucleofector kit (Amaxa) gave a 60\% transfection efficiency. The reduced expression of MMP-2 was checked $48 \mathrm{~h}$ after siRNA transfection by both real-time RT-PCR and zymography. As shown in Fig. 8A, the levels of mRNA coding for MMP-2 were greatly reduced in siRNA transfected young AoSMCs, reaching $\sim 95 \%$ of inhibition. Scrambled siRNA transfected cells confirm the specificity of the silencing
Figure 7. Migration of young and aged AoSMCs on gelatin-coated Transwell apparatus. A) Photograph of migrated young or aged AoSMCs after $6 \mathrm{~h}$ of incubation without adding any modulators in the lower chamber of the Transwell. One representative high-power microscope fields (HPF) is shown (magnification $20 \times$ ). The mean number $\pm \mathrm{SE}$ of cell migrated per $\mathrm{HPF}$ and the significance between young and aged cells is reported. $1 \times 10^{5}$ young AoSMCs $(B)$ or the same amount of aged AoSMCs $(C)$ were seeded on the upper side of the chamber while modulators (i.e., TIMP-1, TIMP-2, GM6001, or active MMP-2) have been added to the lower compartment at the concentration indicated in Materials and Methods. After $6 \mathrm{~h}$ of incubation at $37^{\circ} \mathrm{C}$, migrated cells were stained with Crystal violet and counted. The number of migrated cells per HPF is reported.
A

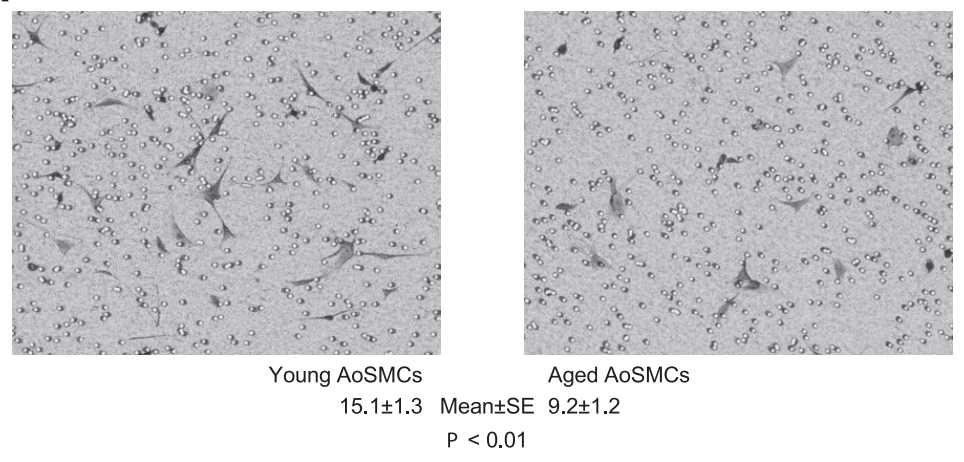

B
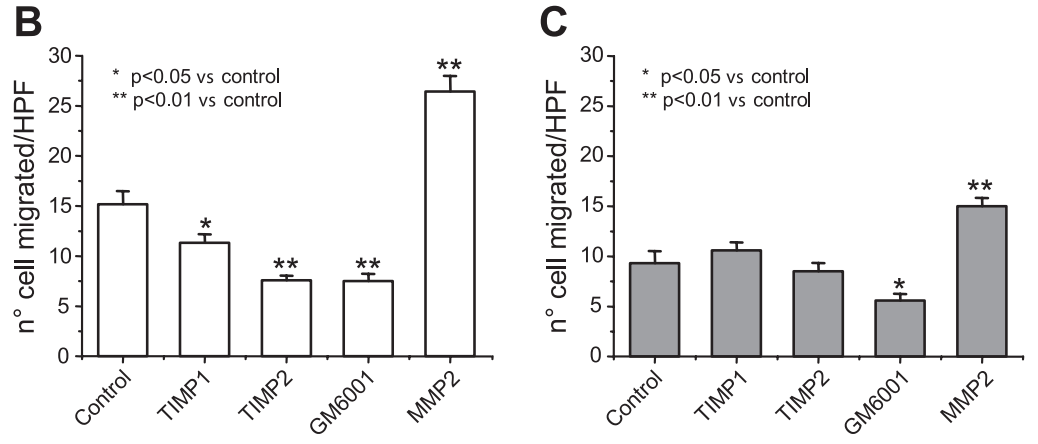
A

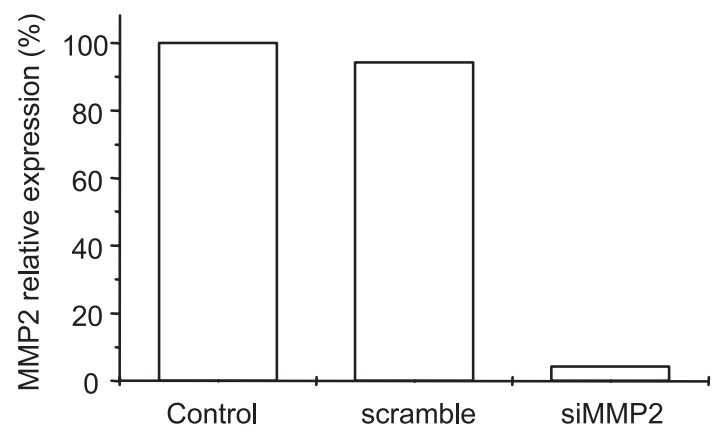

B

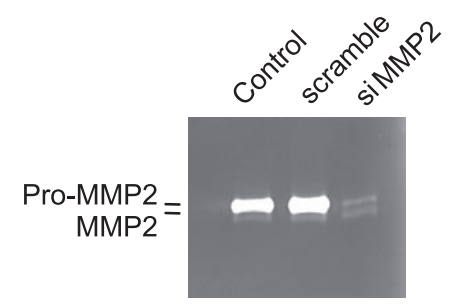

C

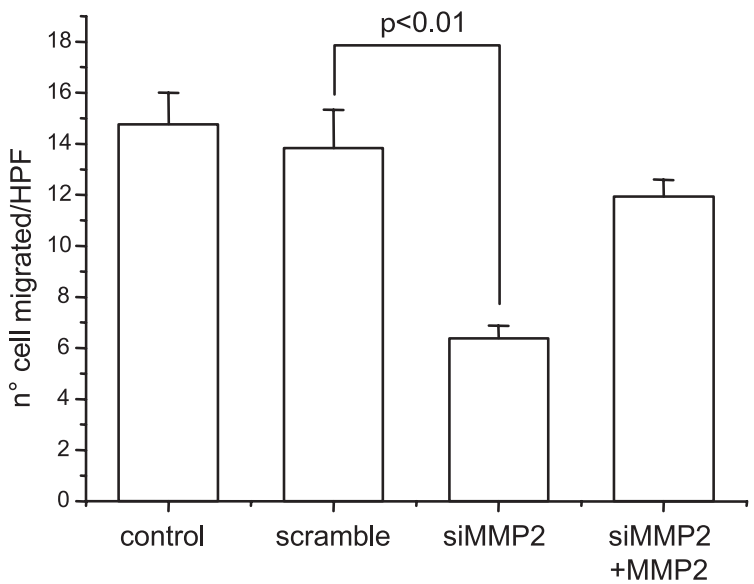

Figure 8. Knockdown of MMP-2 in young AoSMCs. A) Quantification of the transcripts coding for MMP-2 by real-time RTPCR in young cells transfected with $5 \mu \mathrm{g}$ of siRNA against MMP-2 (siMMP-2) or $5 \mu \mathrm{g}$ of scramble siRNA (scramble) after $48 \mathrm{~h}$. The control experiment was performed transfecting the cells with only Nucleofector Solution (Amaxa). Relative expression is reported in percent referred to the control. B) MMP-2 activity in young AoSMCs silenced for MMP-2 by zymography. $10^{6}$ cells were transfected with $5 \mu \mathrm{g}$ of siRNA against MMP-2 (siMMP-2) or $5 \mu \mathrm{g}$ of scramble siRNA (scramble) or with only Nucleofector Solution (control). $2 \mu \mathrm{g}$ of each conditioned cell medium were loaded in the gel. C) Migration of young MMP-2silenced AoSMCs. $1 \times 10^{5}$ cells, transfected with $5 \mu \mathrm{g}$ of siRNA against MMP-2 (siMMP-2) or $5 \mu \mathrm{g}$ of scramble siRNA (scramble) or with only Nucleofector Solution (control), were seeded in the upper chamber of gelatin-coated Transwell apparatus. As control, $0.3 \mu \mathrm{g} / \mathrm{ml}$ active-MMP-2 (Calbiochem) was added to the lower chamber of the Transwell (siMMP-2+MMP-2). After $6 \mathrm{~h}$ of incubation at $37^{\circ} \mathrm{C}$, migrated cells were stained with Crystal violet and counted.

effect. Accordingly, the reduced expression of MMP-2 mRNA, in zymography MMP-2 activity is strongly reduced in conditioned medium of young AoSMC trans- fected with siRNA against MMP-2 compared with the gelatinase activity of either control untransfected or scramble siRNA transfected cells (Fig. $8 B$ ). To better quantify the MMP-2 activity, ELISA tests revealed that the total gelatinase concentration was 4.8, 18.0, and $17.9 \mathrm{ng} / \mathrm{ml}$ in MMP-2 siRNA, scramble siRNA, and control transfected young AoSMCs, respectively. Active MMP-2 was 2.4, 8.8, and $7.8 \mathrm{ng} / \mathrm{ml}$ in MMP-2 siRNA, scramble siRNA, and control transfected young AoSMCs, respectively, corresponding to an active MMP-2 inhibition of $\sim 70 \%$.

MMP-2-silenced young AoSMCs showed a significant decrease in migratory behavior $(P<0.01)$ as clearly shown in Fig. $8 C$. This reduced migration capability was actually due to MMP-2 as the scramble siRNA transfected cells migrated as untransfected young AoSMCs (Fig. 8C). Moreover, we can exclude that the lower extent of migration of MMP-2 siRNA transfected cells could be due to a specific toxic effect of MMP-2 siRNA, since purified active MMP-2 added to these cells restored the normal migratory behavior almost to the concentration of the control (i.e., siRNA scramble) (Fig. 8C).

To demonstrate the pivotal role of MMP-2:TIMP-2 ratio in the control of migration during aging, we silenced the TIMP-2 expression in aged AoSMC by siRNA. Using the same conditions of young AoSMC, we obtained a transfection efficiency of $\sim 55 \%$ (result not shown). The reduced expression of TIMP-2 was checked $48 \mathrm{~h}$ after siRNA transfection by real-time RT-PCR and Western blot. As shown in Fig. 9A, the levels of mRNA coding for TIMP-2 were greatly reduced in TIMP-2 siRNA transfected aged AoSMCs reaching $\sim 80 \%$ of inhibition, whereas scrambled siRNA transfected cells showed a TIMP-2 expression concentration similar to untransfected cells. The reduced expression of TIMP-2 was also confirmed detecting $21 \mathrm{kDa}$ TIMP-2 protein in the conditioned culture medium of siRNA transfected cells by means of Western blot (Fig. 9B). We have determined an activation of MMP-2 in the conditioned medium of aged AoSMCs transfected with siRNA against TIMP-2 (result not shown).

TIMP-2-silenced aged AoSMCs showed a significant increase in migratory behavior $(P<0.01)$ as clearly shown in Fig. 9C. The specificity of this latter result was supported by evidence that the migration of scramble siRNA transfected and untransfected cells were comparable (Fig. 9C). Moreover, the addition of human purified TIMP-2 to aged TIMP-2 silenced cells, which determined a reduction of migration to the control levels (Fig 9B), confirmed that the fine balancing of MMP-2 and TIMP-2 was critical in controlling the migration during in vitro aging of AoSMC.

\section{DISCUSSION}

Aging is a complex and multifactorial process that involves changes in every part of the organism. Such adaptations correspond to dramatic modifications at 


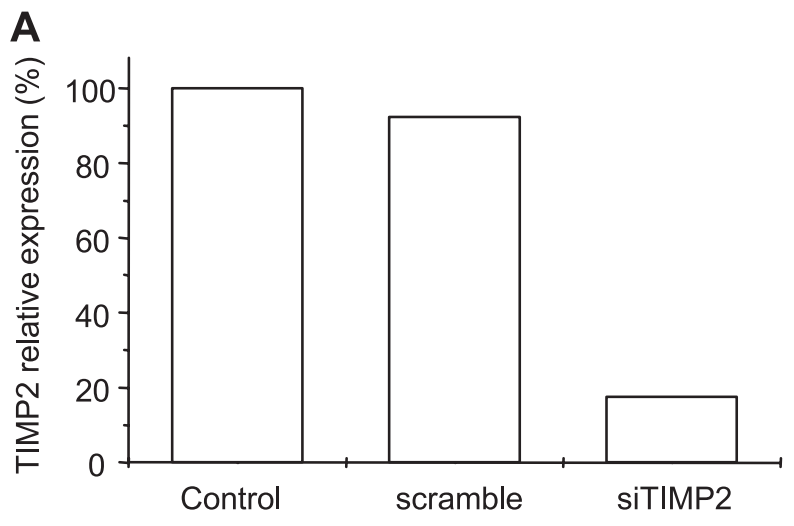

B

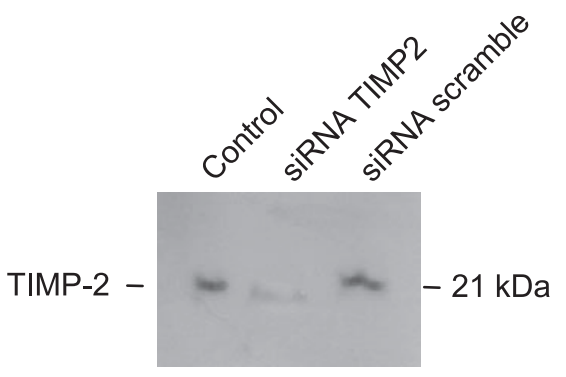

C

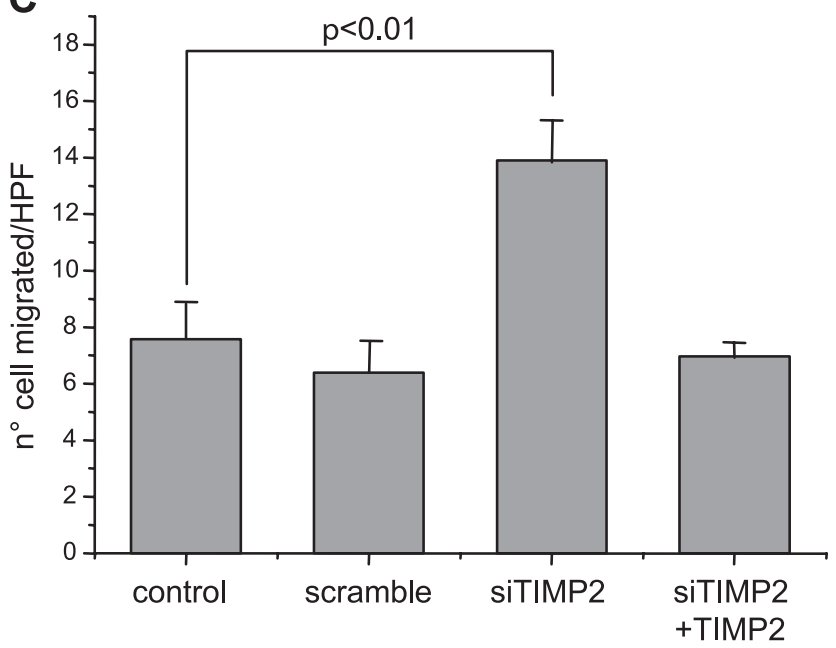

Figure 9. Knockdown of TIMP-2 in aged AoSMCs. A) Quantification of the transcripts coding for TIMP-2 by real-time RT-PCR in aged cells transfected with $5 \mu \mathrm{g}$ of siRNA against TIMP-2 (siTIMP2) or $5 \mu \mathrm{g}$ of scramble siRNA (scramble) after $48 \mathrm{~h}$. The control experiment (control) was performed transfecting the cells with only Nucleofector Solution (Amaxa). Relative expression is reported in percent referred to the control. $B$ ) Western blot analysis on $10 \times$ concentrated conditioned medium of aged AoSMCs transfected with $5 \mu \mathrm{g}$ of siRNA against TIMP-2 (siTIMP2) or $5 \mu \mathrm{g}$ of scramble siRNA (scramble) after $48 \mathrm{~h}$ using anti-TIMP-2 antibodies. C) Migration of aged TIMP-2-silenced AoSMCs. $1 \times 10^{5}$ cells, transfected with $5 \mu \mathrm{g}$ of siRNA against TIMP-2 or $5 \mu \mathrm{g}$ of scramble siRNA or with only Nucleofector Solution, were seeded in the upper chamber of gelatin-coated Transwell apparatus. As control, $0.3 \mu \mathrm{g} / \mathrm{ml}$ purified TIMP-2 (Calbiochem) was added to the lower chamber of the Transwell (siTIMP2+TIMP2). After $6 \mathrm{~h}$ of incubation at $37^{\circ} \mathrm{C}$, migrated cells were stained with Crystal violet and counted. molecular concentration such as cellular metabolism, tissue compositions, DNA damage, and many others (for a review, see ref 31). Even though the mechanism of aging is far from being understood, it is well established that cellular senescence occurs in vivo and in vitro. Therefore, cellular senescence may be a useful tool to study aging processes (29). As aging is a major risk for cardiovascular disease, which represents the most important cause of death in western countries, we have developed a cellular system to study age-related modifications in AoSMC, critical components of the vascular wall. A large body of evidence supports the in vitro aging model; for instance, the comparison of telomere lengths between human endothelial cells aged in vitro and those obtained from donors of different ages do not show any difference (32).

As AoSMCs play a pivotal role in both physiological and pathological processes that occur in vascular system, we set up an in vitro aging model based on sequential cell passages (population doublings) in vitro. Moreover, the relationship between in vitro and in vivo aging was described by Martin et al. (33), who reported that replicative capabilities of human SMC cultures decline with donor aging; in our cultures we obtained a similar growth decrease during progressive cell passages (results not shown). In fact, in our aged AoSMC cultures we detected the senescent phenotype visualizing both SA $\beta$-Gal activity, which is proposed as an effective marker of cell senescence as telomere length $(24,28,43)$, and cyclin-dependent inhibitor p16 mRNA, which was recently demonstrated to accumulate in senescent cells (25). Moreover, to exclude AoSMC dedifferentiation events, we have ruled out such phenomena detecting the same expression and cellular distribution of the typical AoSMC markers (i.e., $\alpha$-SMA and calponin) in young as well as in aged cells. In our model, we found other aspects typical of aged AoSMCs such as increased cell size and trypsin digestion resistance (35). Hence, the simultaneous expression of senescent markers and SMC phenotypical proteins indicates that controlled sequential passages in vitro can be considered a valid model to study age-related modifications in AoSMCs.

In this work, we describe a clear regulation of MMP-2 activity during the aging process, and how such regulation affects the migration of AoSMCs. Only in conditioned culture medium of young AoSMCs did we find the active form of MMP-2. On the other hand, inactive pro-MMP-2 was present at about the same amount in young and aged cell conditioned medium by means of gel zymography, ELISA quantification, and Western blot analysis. This result is strictly related to the quantification of MMP-2 mRNA performed with real-time RT-PCR. In fact, no significant differences were detected between the MMP-2 transcription concentration in young and aged AoSMCs. The related gelatinase MMP-9 was not detected in our cell model, in agreement with a previous study reporting that, in mouse SMC, MMP-9 expression is present only after injury or inflammatory stimulation (11). 
MMP activity is implicated in various vascular diseases like hypertension, arterial restenosis, atherosclerosis, and aortic aneurismal disease (36). Because there is a direct correlation between aging and the development of circulatory diseases, some studies raised the hypothesis that aging could cause a MMPs activation, then pathology onset (37). Other authors indicate in the inflammatory process occurring in aged vascular tissue the trigger that activates the MMPs (38). To avoid the effect of inflammatory stimuli and to simplify the model, AoSMCs are cultured in vitro without the complex and not yet fully identified signals deriving from the surroundings cells that are present in vivo. This in vitro aging system could be considered a not pathological aging model as lacking the signaling deriving from other cells. From another point if view, our model can more carefully identify the real role of AoSMCs in aging process. Moreover, a recent paper reported that an increase of MMP-2 activity can be detectable in healthy human aorta specimens from donors of different ages (37). Such an MMP-2 increase was detected mainly in the aorta intima layer where AoSMCs are not present, highlighting a peculiar spatio-temporal specialization of each cells types populating the vessel walls. The data about MMPs activation were often obtained from whole tissue specimens, and therefore lacked the specific role of a single cell type in pathology onset. However, comparing the gene expression of MMPs and their regulators in fetal and aged ( $>70$ years old) human aorta samples, we detected an evident agreement between results obtained in our in vitro model and in vivo specimens. This finding could demonstrate that the MMP-2 regulation observed in vitro could also be present in vivo during natural aging, even if further investigation must be done to elucidate this point at a functional concentration.

Activation of the pro-MMP-2 in MMP-2 is achieved by several mechanisms involving other proteins (i.e., other MMPs, thrombin, and plasmin) (39). MT1-MMP plays a crucial role in pro-MMP-2 activation, and we have found an increase of MT1-MMP mRNA and protein in young AoSMCs in accordance with the elevated activation of pro-MMP-2 detected in such cells. Even if it were contradictory, MT1-MMP pro-MMP-2 activation mechanism involves the specific MMP-2 inhibitor TIMP-2. In fact, TIMP-2 is necessary as a docking element between pro-MMP-2 and MT1-MMP, thus allowing the cleavage of the propeptide of pro-MMP-2 by MT1-MMP $(8,40$, $41)$. Based on this consideration, we have visualized a higher TIMP-2 activity in total protein extracts prepared from young than aged AoSMCs. Cell-associated TIMP-2 represents the intracellular and MT1-MMPbound inhibitor. The intracellular TIMP-2, which is associated with lysosomes and endosomes fractions, is destined to a rapid degradation (42) whereas membrane-associated TIMP-2 represents the major fraction of cellular TIMP-2, as clearly shown by confocal immunolocalization both on cultured cells and in vivo (43$45)$. Therefore, although we could not directly demonstrate the real MT1-MMP/TIMP-2/pro-MMP-2 complex formation, MT1-MMP and TIMP2 could be expressed more in membrane of young AoSMC than in that of aged cells, indicating that pro-MMP-2 activation complex (i.e., MT1-MMP, TIMP2, and pro-MMP-2) may be more abundant in young cells, leading therefore to more efficient activation of pro-MMP-2.

As TIMPs play a critical role not only in the modulation of MMP-2 activity, but also in the conversion from inactive to active form of the enzyme, we have focused our attention on TIMP expression in young and aged AoSMCs by means of quantitative real-time RT-PCR, reverse zymography, and Western blot analyses. We found elevated TIMP-1 and TIMP-2 mRNA expression and activity in aged AoSMCs, thus supporting the hypothesis of an inhibition of MMP-2 activation in aged cells, which could also depend on an up-regulation of TIMP messengers. Although TIMP-1 and TIMP-2 have a wide range of substrates and can also be active on MMP-2, the specific inhibitor of MMP-2 is TIMP-2 (46). TIMP-2 inhibitory activity was found to be higher in the conditioned cell culture medium of aged than of young AoSMCs, confirming the quantification of TIMP-2 mRNA performed by real-time RT-PCR. The fine regulation of activators and inhibitors of MMP activities has been demonstrated to play a pivotal role in the onset of different pathologies of the vascular system (36) as well as tumor growth and invasion (47), osteoarthritis (48), and recently in plasma of women who subsequently developed preeclampsia (49). Therefore, a large body of evidence suggests that the unbalance between MMPs and TIMPs is critical in the pathophysiology not only in the vascular system, but also in other tissues.

In the postgenomic era, the scientific community is trying to answer the question "how genes are regulated" as the gene identification step has been reported (50, 51). However, from a physiological point of view it would be important to study cell behavior in correlation with cell gene expression. As the migration of vascular SMCs plays an important role in the production of the thickened intima after vascular injury (52) and also in the neovascularization that occurs during wound healing, ischemia, postinterventional restenosis and the formation of advanced atherosclerotic plaques, we investigated whether the different activity of MMP-2 in young and aged AoSMCs affects cell migration properties. By means of modified Boyden chamber assays using Transwell apparatus coated with gelatin, in order to have a micro-environment resembling vascular basal membrane (53), we measured higher migration capabilities in young in comparison to aged cells (Fig. 4). These data are in agreement with Ruiz-Torres et al. (54), who describe that human SMCs from old donor possess a lower migration rate than SMCs from young donor. Nevertheless, contradictory results have been obtained comparing the migratory behavior of SMCs from young or aged rat aorta (19) indicating that a probable species-specific differences in the aging process could exist (54). In fact, aging may be an atypical biological process, because no genes appear to have evolved specifically to cause it (55). 
Indeed, because age-related changes are the unprogrammed results of stringent selection for early reproductive process, aging may be an example of a biological phenomenon where there is a low concentration of mechanistic conservation between diverse metazoans (56).

The decreased MMP-2 activity and increased TIMP-1 and TIMP-2 expression address the question of whether MMP-2 affects cell migration in our system. Hence, human purified TIMP-1 and TIMP-2 were added to young AoSMCs to mimic the TIMP expression concentration of aged cells. As proposed in our hypothesis, young AoSMCs treated with TIMP-2 migrated as aged cells, whereas TIMP-1-treated cells showed only a $25 \%$ of migration inhibition. This result could be justified considering the nonspecific effect of TIMP-1 on MMP-2 (15). We also treated aged AoSMCs with either TIMP-1, TIMP-2, or human active purified MMP-2. TIMP treatments did not further inhibit the migration of aged AoSMCs. This could be explained considering that MMP and TIMP complexes are in a 1:1 M ratio (15); an extra addition of TIMP to cells that already expresses such proteins could not produce any more MMP inhibition. Human active purified MMP-2 was added to aged AoSMCs to mimic young cell behavior and, as expected, the migration of aged cells reached the concentration observed in young cells.

When we inhibited the gelatinase activities of young AoSMCs using GM6001, also known to inhibit a wide range of proteases, including the ADAM family, we found a slower migration similar to results obtained with TIMP-2-treated cells. This indicates that MMP-2/ TIMP-2 were the major proteins involved in migration control of AoSMC. However, this was an indirect evidence, and we have specifically demonstrated the pivotal role of MMP-2 and TIMP-2 silencing MMP-2 by siRNA in young cells and TIMP-2 in aged cells. Both transfections reached a very high yield of inhibition decreasing the presence of MMP-2 and TIMP-2 transcripts of the $95 \%$ and $80 \%$, respectively. Such a difference in transfection efficiency could be explained considering the difficulty of aged (high PD) cells to be transfected efficiently. siRNA MMP-2-silenced young AoSMCs showed decreased migratory properties that could be recovered by adding human active purified MMP-2. By contrast, TIMP-2-silenced aged AoSMCs increased migratory properties that could be reverted by adding human recombinant TIMP-2. In this way, we were able to obtain in young cells the MMP-2 inhibition typical of aged AoSMCs and in aged cells the typical MMP-2 activation of young AoSMCs. These experiments support the pivotal role of the ratio MMP-2: TIMP-2 to control the migration of AoSMC during in vitro aging. The lower MMP-2 activity, and then the lower migration capability of aged cells reported in in vivo studies, could be correlated with the minor presence of SMC in atheromatous plaque from older patient carotids (57). In such "aged plaque" decreased MMP-2 activity, an increase of MMP-9 activity and CD68 positive cells clearly demonstrates that other cells (i.e., macrophages) could modify the behavior of resident cells modulating the ECM composition through inflammatory process. The central role of MMP-2 in the pathophysiology of vascular system was recently confirmed in MMP-2 knockout (KO) mice (6). In fact AoSMCs from MMP-2 KO mice showed limited migratory capabilities; moreover, the MMP-2 KO mice showed a very limited intimal thickening during carotid artery injury, indicating the critical role this enzyme plays in the physiopathology of blood vessels in vivo.

In conclusion, we have reported an inhibition of MMP-2 activity during sequential AoSMC culture passages that could originate from an increase of TIMP-1 and TIMP-2 or a decrease of MT1-MMP. We also demonstrated that during in vitro aging the AoSMC migration decreases and such cellular behavior can be strictly regulated by MMP-2 and TIMP-2 activities. [ F

We thank Claudio Monetti and Edward Maytin for a critical review of the manuscript, and Raffaella Cinquetti, Andrea Moriondo, Annalisa Grimaldi, and Luisa Guidali for technical assistance. The authors gratefully acknowledge the "Centro Grandi Attrezzature per la Ricerca Biomedica" Università degli Studi dell'Insubria for instruments availability. This work was supported by COFIN to D.V. and CIB to A.P.

\section{REFERENCES}

1. Ross, R. (1993) The pathogenesis of atherosclerosis: a perspective for the 1990s. Nature 362, 801-809

2. Halayko, A. J., and Solway, J. (2001) Molecular mechanisms of phenotypic plasticity in smooth muscle cells. J. Appl. Physiol 90, 358-368

3. Sperti, G., van Leeuwen, R. T., Quax, P. H., Maseri, A., and Kluft, C. (1992) Cultured rat aortic vascular smooth muscle cells digest naturally produced extracellular matrix. Involvement of plasminogen-dependent and plasminogen-independent pathways. Circ. Res. 71, 385-392

4. Pauly, R. R., Passaniti, A., Bilato, C., Monticone, R., Cheng, L., Papadopoulos, N., Gluzband, Y. A., Smith, L., Weinstein, C., and Lakatta, E. G. (1994) Migration of cultured vascular smooth muscle cells through a basement membrane barrier requires type IV collagenase activity and is inhibited by cellular differentiation. Circ. Res. 75, 41-54

5. Passi, A., Negrini, D., Albertini, R., Miserocchi, G., and De Luca, G. (1999) The sensitivity of versican from rabbit lung to gelatinase A (MMP-2) and B (MMP-9) and its involvement in the development of hydraulic lung edema. FEBS Lett. 456, 93-96

6. Johnson, C., and Galis, Z. S. (2004) Matrix metalloproteinase-2 and -9 differentially regulate smooth muscle cell migration and cell-mediated collagen organization. Arterioscler. Thromb. Vasc. Biol. 24, 54-60

7. Kuzuya, M., Kanda, S., Sasaki, T., Tamaya-Mori, N., Cheng, X. W., Itoh, T., Itohara, S., and Iguchi, A. (2003) Deficiency of gelatinase A suppresses smooth muscle cell invasion and development of experimental intimal hyperplasia. Circulation 108, $1375-1381$

8. Sternlicht, M. D., and Werb, Z. (2001) How matrix metalloproteinases regulate cell behavior. Annu. Rev. Cell Dev. Biol. 17, 463-516

9. Iozzo, R. V. (1998) Matrix proteoglycans: from molecular design to cellular function. Annu. Rev. Biochem. 67, 609-652

10. Forsten, K. E., Courant, N. A., and Nugent, M. A. (1997) Endothelial proteoglycans inhibit bFGF binding and mitogenesis. J. Cell. Physiol. 172, 209-220

11. Whatling, C., McPheat, W., and Hurt-Camejo, E. (2004) Matrix management: assigning different roles for MMP-2 and 
MMP-9 in vascular remodeling. Arterioscler. Thromb. Vasc. Biol. 24, $10-11$

12. Galis, Z. S., Muszynski, M., Sukhova, G. K., Simon-Morrissey, E., Unemori, E. N., Lark, M. W., Amento, E., and Libby, P. (1994) Cytokine-stimulated human vascular smooth muscle cells synthesize a complement of enzymes required for extracellular matrix digestion. Circ. Res. 75, 181-189

13. Seiki, M. (2003) Membrane-type 1 matrix metalloproteinase: a key enzyme for tumor invasion. Cancer Lett. 194, 1-11

14. Baker, A. H., Edwards, D. R., and Murphy, G. (2002) Metalloproteinase inhibitors: biological actions and therapeutic opportunities. J. Cell Sci. 115, 3719-3727

15. Lambert, E., Dasse, E., Haye, B., and Petitfrere, E. (2004) TIMPs as multifacial proteins. Crit. Rev. Oncol. Hematol. 49, 187-198

16. Cheng, X. W., Kuzuya, M., Sasaki, T., Kanda, S., Tamaya-Mori, N., Koike, T., Maeda, K., Nishitani, E., and Iguchi, A. (2004) Green tea catechins inhibit neointimal hyperplasia in a rat carotid arterial injury model by TIMP-2 overexpression. Cardiovasc. Res. 62, 594-602

17. Cheng, L., Mantile, G., Pauly, R., Nater, C., Felici, A., Monticone, R., Bilato, C., Gluzband, Y. A., Crow, M. T., StetlerStevenson, W., and Capogrossi, M. C. (1998) Adenovirus-mediated gene transfer of the human tissue inhibitor of metalloproteinase-2 blocks vascular smooth muscle cell invasiveness in vitro and modulates neointimal development in vivo. Circulation 98, 2195-2201

18. Kadoglou, N. P., and Liapis, C. D. (2004) Matrix metalloproteinases: contribution to pathogenesis, diagnosis, surveillance and treatment of abdominal aortic aneurysms. Curr. Med. Res. Opin. 20, 419-432

19. Li, Z., Cheng, H., Lederer, W. J., Froehlich, J., and Lakatta, E. G. (1997) Enhanced proliferation and migration and altered cytoskeletal proteins in early passage smooth muscle cells from young and old rat aortic explants. Exp. Mol. Pathol. 64, 1-11

20. McCaffrey, T. A., Nicholson, A. C., Szabo, P. E., Weksler, M. E., and Weksler, B. B. (1988) Aging and arteriosclerosis. The increased proliferation of arterial smooth muscle cells isolated from old rats is associated with increased platelet-derived growth factor-like activity. J. Exp. Med. 167, 163-174

21. Lundberg, M. S., and Crow, M. T. (1999) Age-related changes in the signaling and function of vascular smooth muscle cells. Exp. Gerontol. 34, 549-557

22. Katsuda, S., and Kaji, T. (2003) Atherosclerosis and extracellular matrix. J. Atheroscler. Thromb. 10, 267-274

23. Li, Z., Froehlich, J., Galis, Z. S., and Lakatta, E. G. (1999) Increased expression of matrix metalloproteinase-2 in the thickened intima of aged rats. Hypertension 33, 116-123

24. Dimri, G. P., Lee, X., Basile, G., Acosta, M., Scott, G., Roskelley, C., Medrano, E. E., Linskens, M., Rubelj, I., and Pereira-Smith, O. (1995) A biomarker that identifies senescent human cells in culture and in aging skin in vivo. Proc. Natl. Acad. Sci. USA 92 , 9363-9367

25. Wang, W., Martindale, J. L., Yang, X., Chrest, F. J., and Gorospe, M. (2005) Increased stability of the p16 mRNA with replicative senescence. EMBO Rep. 6, 158-164

26. Oliver, G. W., Leferson, J. D., Stetler-Stevenson, W. G., and Kleiner, D. E. (1997) Quantitative reverse zymography: analysis of picogram amounts of metalloproteinase inhibitors using gelatinase A and B reverse zymograms. Anal. Biochem. 244, 161-166

27. Mazzieri, R., Masiero, L., Zanetta, L., Monea, S., Onisto, M., Garbisa, S., and Mignatti, P. (1997) Control of type IV collagenase activity by components of the urokinase-plasmin system: a regulatory mechanism with cell-bound reactants. EMBO J. $\mathbf{1 6}$, 2319-2332

28. van der, L. B., Fenton, M. J., and Erusalimsky, J. D. (1998) Cytochemical detection of a senescence-associated beta-galactosidase in endothelial and smooth muscle cells from human and rabbit blood vessels. Exp. Cell Res. 241, 309-315

29. Campisi, J. (2005) Senescent cells, tumor suppression, and organismal aging: good citizens, bad neighbors. Cell 120, 513522

30. Liu, S. Q., and Fung, Y. C. (1998) Changes in the organization of the smooth muscle cells in rat vein grafts. Ann. Biomed. Eng 26, 86-95
31. Troen, B. R. (2003) The biology of aging. Mt. Sinai J. Med. 70, 3-22

32. Chang, E., and Harley, C. B. (1995) Telomere length and replicative aging in human vascular tissues. Proc. Natl. Acad. Sci. USA 92, 11190-11194

33. Martin, G. M., Ogburn, C. E., and Wight, T. N. (1983) Comparative rates of decline in the primary cloning efficiencies of smooth muscle cells from the aging thoracic aorta of two murine species of contrasting maximum life span potentials. Am. J. Pathol. 110, 236-245

34. Kim, H., Farris, J., Christman, S. A., Kong, B. W., Foster, L. K., O'Grady, S. M., and Foster, D. N. (2002) Events in the immortalizing process of primary human mammary epithelial cells by the catalytic subunit of human telomerase. Biochem. J. 365, 765-772

35. Passi, A., Albertini, R., Campagnari, F., and De Luca, G. (1997) Modifications of proteoglycans secreted into the growth medium by young and senescent human skin fibroblasts. FEBS Lett. 402, 286-290

36. Lijnen, H. R. (2003)/2004) Metalloproteinases in development and progression of vascular disease. Pathophysiol. Haemost. Thromb. 33, 275-281

37. McNulty, M., Spiers, P., McGovern, E., and Feely, J. (2005) Aging is associated with increased matrix metalloproteinase-2 activity in the human aorta. Am. J. Hypertens. 18, 504-509

38. Newby, A. C. (2005) Dual role of matrix metalloproteinases (matrixins) in intimal thickening and atherosclerotic plaque rupture. Physiol. Rev. 85, 1-31

39. Galis, Z.S., Kranzhofer, R., Fenton, J. W., 2nd, and Libby, P. (1997) Thrombin promotes activation of matrix metalloproteinase- 2 produced by cultured vascular smooth muscle cells. Arterioscler. Thromb. Vasc. Biol. 17, 483-489

40. Sounni, N. E., Janssen, M., Foidart, J. M., and Noel, A. (2003) Membrane type-1 matrix metalloproteinase and TIMP-2 in tumor angiogenesis. Matrix Biol. 22, 55-61

41. Worley, J. R., Thompkins, P. B., Lee, M. H., Hutton, M., Soloway, P., Edwards, D. R., Murphy, G., and Knauper, V. (2003) Sequence motifs of tissue inhibitor of metalloproteinases 2 (TIMP-2) determining progelatinase A (proMMP-2) binding and activation by membrane-type metalloproteinase 1 (MT1MMP). Biochem. J. 372, 799-809

42. Maquoi, E., Frankenne, F., Baramova, E., Munaut, C., Sounni, N. E., Remacle, A., Noel, A., Murphy, G., and Foidart, J.-M. (2000) Membrane type I matrix metalloproteinase-associated degradation of tissue inhibitor of metalloproteinase 2 in human tumor cell lines. J. Biol. Chem., 275, 11368-11378

43. Nour, N., Mayer, G., Mort, J. S., Salvas, A., Mbikay, M., Morrison, C. J., Overall, C. M., and Seidah, N. G. (2005) The cysteine-rich domain of the secreted proprotein convertases pc5a and pace 4 functions as a cell surface anchor and interacts with tissue inhibitors of metalloproteinases. Mol. Biol. Cell 16, 5215-5226

44. Pérez-Martínez, L., and Jaworski, D. M. (2005) Tissue inhibitor of etalloproteinase-2 promotes neuronal differentiation by acting as an anti-mitogenic signal. J. Neurosci. 25, 4917-4929

45. Hoegy, S. E., Oh, H. R., Corcoran, M.L., and Stetler-Stevenson, W. G. (2001) Tissue inhibitor of metalloproteinases-2 (timp-2) suppresses tkr-growth factor signaling independent of metalloproteinase inhibition. J. Biol. Chem. 276, 3203-3214

46. Howard, E. W., and Banda, M. J. (1991) Binding of tissue inhibitor of metalloproteinases 2 to two distinct sites on human 72-kDa gelatinase. Identification of a stabilization site. J. Biol. Chem. 266, 17972-17977

47. Giannelli, G., and Antonaci, S. (2002) Gelatinases and their inhibitors in tumor metastasis: from biological research to medical applications. Histol. Histopathol. 17, 339-345

48. Iannone, F., and Lapadula, G. (2003) The pathophysiology of osteoarthritis. Aging Clin. Exp. Res. 15, 364372

49. Myers, J. E., Merchant, S. J., Macleod, M., Mires, G. J., Baker, P. N., and Davidge, S. T. (2005) MMP-2 levels are elevated in the plasma of women who subsequently develop preeclampsia. Hypertens. Pregnancy 24, 103-115

50. Lander, E. S., Linton, L. M., Birren, B., Nusbaum, C., Zody, M. C., Baldwin, J., Devon, K., Dewar, K., Doyle, M., FitzHugh, W., et al. (2001) Initial sequencing and analysis of the human genome. Nature 409, 860-921

51. Waterston, R. H., Lindblad-Toh, K., Birney, E., Rogers, J., Abril, J. F., Agarwal, P., Agarwala, R., Ainscough, R., Alexandersson, 
M., An, P., et al. (2002) Initial sequencing and comparative analysis of the mouse genome. Nature 420, 520-562

52. Gibbons, G. H., and Dzau, V. J. (1994) The emerging concept of vascular remodeling. N. Engl. J. Med. 330, 1431-1438

53. Kanda, S., Kuzuya, M., Ramos, M. A., Koike, T., Yoshino, K., Ikeda, S., and Iguchi, A. (2000) Matrix metalloproteinase and alphavbeta3 integrin-dependent vascular smooth muscle cell invasion through a type I collagen lattice. Arterioscler. Thromb. Vasc. Biol. 20, 998-1005

54. Ruiz-Torres, A., Lozano, R., Melon, J., and Carraro, R. (2003) Age-dependent decline of in vitro migration (basal and stimulated by IGF-1 or insulin) of human vascular smooth muscle cells. J. Gerontol. A Biol. Sci. Med. Sci. 58, B1074-B1077
55. Partridge, L., and Gems, D. (2002) The evolution of longevity. Curr. Biol. 12, R544-R546

56. Kipling, D., Davis, T., Ostler, E. L., and Faragher, R. G. (2004) What can progeroid syndromes tell us about human aging? Science 305, 1426-1431

57. van Oostrom, O., Velema, E., Schoneveld, A.H., de Vries, J. P., de Bruin, P., Seldenrijk, C. A., de Kleijn, D. P., Busser, E., Moll, F. L., Verheijen, J. H., Virmani, R., and Pasterkamp, G. (2005) Agerelated changes in plaque composition: a study in patients suffering from carotid artery stenosis. Cardiovasc. Pathol. 14, 126-134

Received for publication June 23, 2005. Accepted for publication February 10, 2006. 